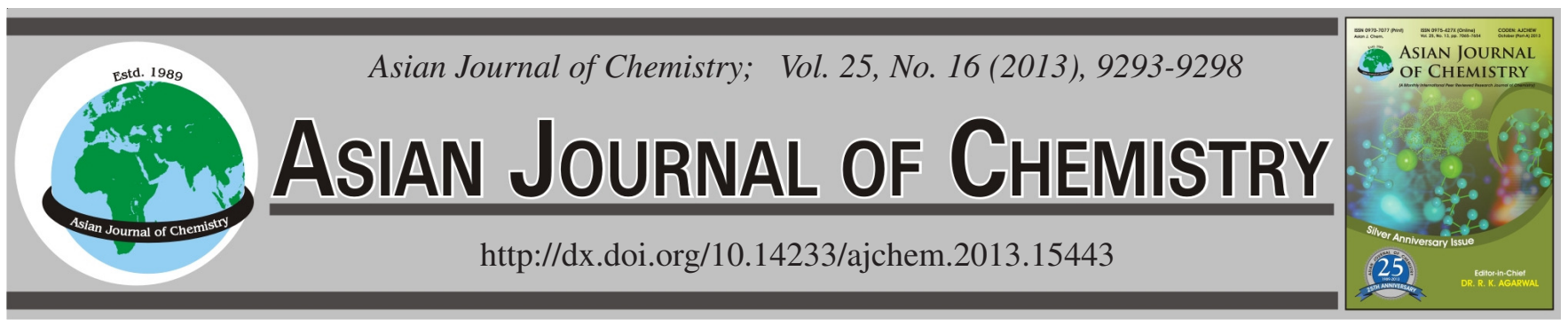

\title{
Synthesis and Structure Activity Relationship of Rigidized Indolyl Pyrrolidine Derivatives as 5-HT6 Receptor Ligands
}

\author{
Ramakrishna Nirogi $^{1, *}$, Adireddy Dwarampudi ${ }^{1,2}$, Venugopalarao Bhatta $^{1}$, Laxman Kota $^{1}$ and P.K. Dubey ${ }^{2}$
}

${ }^{1}$ Discovery Research, Suven Life Sciences Ltd., Serene Chambers, Road-5, Avenue-7, Banjara Hills, Hyderabad-500 034, India ${ }^{2}$ Department of Chemistry, Jawaharlal Nehru Technological University Hyderabad, Kukatpally, Hyderabad-500 085, India

*Corresponding author: Fax: +91 40 23541152; Tel: +91 40 23556038/23541142; E-mail: nvsrk@suven.com

(Received: 7 March 2013;

Accepted: 20 September 2013)

AJC-14164

A novel series of rigidized indolyl pyrrolidine derivatives have been designed by constraining the tryptamine nitrogen through $\alpha$ and $\beta$ carbons. All the synthesized derivatives have shown moderate affinities at 5- $\mathrm{HT}_{6} \mathrm{R}$ when tested in in vitro binding assay. Synthesis, structure activity relationship (SAR), pharmacokinetic profile and in vivo efficacy of a selected compound is the subject matter of this communication.

Key Words: 5-HT 6 R, Structure activity relationship, Pharmacokinetic, Brain penetration, Water maze, Cognition.

\section{INTRODUCTION}

Serotonin (5-hydroxytryptamine, 5-HT) plays an important role in the modulation of neuropsychiatric disorders and exerts its actions via fourteen classes of receptors, which are involved in controlling an array of physiological functions and promoting pathophysiological conditions in states of excess or deficiency. 5- $\mathrm{HT}_{6} \mathrm{R}$ was identified in 1990 in rat and it has become a promising target for the treatment of neuropsychological disorders like anxiety, depression, schizophrenia, epilepsy, obesity, abnormal feeding behaviour and cognitive dysfunctions ${ }^{1-7}$.

Scientists from industries and academia evinced enormous interest in the development of newer ligands for $5-\mathrm{HT}_{6}$ receptors, which have the capability to address debilitating CNS related disorders, hitherto a highly unmet clinical field. There are large number of other publications as well as patents in the past two decades disclosing variety of scaffolds for 5-HT 6 ligands including N-phenylsulfonyl tryptamine $(\mathrm{MS}-245)^{8}$ and 2-phenyltryptamine (PMDT) ${ }^{9}$, which demonstrate the potential of 5- $\mathrm{HT}_{6} \mathrm{R}$ ligands. A number of $5-\mathrm{HT}_{6}$ receptor antagonists like SB-742457 ${ }^{10}$, LuAE58054 ${ }^{11}$, SUVN-502 ${ }^{12}$ and SAM-760 ${ }^{13}$ have entered clinical development.

In our recent publication ${ }^{14}$ we have disclosed the effects of rigidization of phenyl sulfonyl ring in MS-245 through C-2 of indole (Fig. 1). This series of compounds 1 have shown moderate binding affinities towards the $5-\mathrm{HT}_{6} \mathrm{R}$ and were found to be selective ligands towards $5-\mathrm{HT}_{6} \mathrm{R}$. In continuation to this effort, we thought of constraining the tryptamine terminal nitrogen by its incorporation into a five membered pyrrolidine ring through $\alpha$ and $\beta$ carbons. The results of these efforts are the subject matter of this paper.

$\frac{\text { EXPERIMENTAL }}{\text { Infrared spectra were recorded on } \mathrm{KBr} \text { disc and in the }}$
solid state using Perkin-Elmer model 1600 FT-IR spectrophotometer (Perkin-Elmer, Norwalk, CT, USA). Electron spray ionization mass spectra were recorded on a API 4000 triple quadruple instrument (MDS-SCIEX, Concord, Ontario, Canada). ${ }^{1} \mathrm{H}$ NMR spectra were obtained on a Bruker proton NMR spectrometer (Fallanden, Switzerland) at $400 \mathrm{MHz}$. Deuterated reagents were used as solvents and were commercially procured. Tetramethylsilane (TMS) was used as internal standard. Chemical shift values were expressed in parts per million $(\delta)$ and coupling constants were expressed in Hertz (Hz). Column chromatography was performed using 60-120 mesh silica gel and executed under nitrogen pressure (flash chromatography) conditions. All the reagents and solvents used were of reagent grade. Various substituted indoles were synthesized in-house using literature reported procedures ${ }^{15}$ and analytically characterized them thoroughly using spectral data. 2-Bromobenzenesulfonyl chloride was synthesized in-house from 2-bromoaniline via diazonium salt intermediate ${ }^{16}$.

3-(1H-Indol-3-yl)-1-methyl pyrrolidine-2,5-dione (5a): A mixture of indole (4a, $2 \mathrm{~g}, 17.09 \mathrm{mmol})$ and maleic acid (2 $\mathrm{g}, 17.24 \mathrm{mmol}$ ) were heated to $50{ }^{\circ} \mathrm{C}$ and stirred until the mass solidified. The mixture was allowed to stand for $0.5 \mathrm{~h}$ and then diluted with a solution of potassium hydroxide $(28.76 \mathrm{~g}$, $51.27 \mathrm{mmol}$ ) in $50 \mathrm{~mL}$ of water. The reaction mass was heated to $80{ }^{\circ} \mathrm{C}$ and stirred for $0.5 \mathrm{~h}$. The mass was cooled to room 


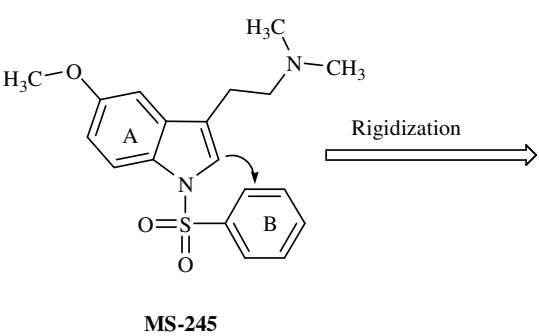

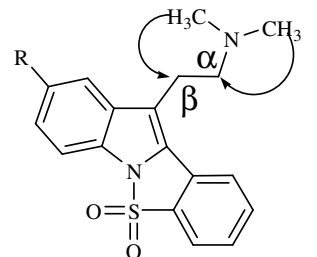
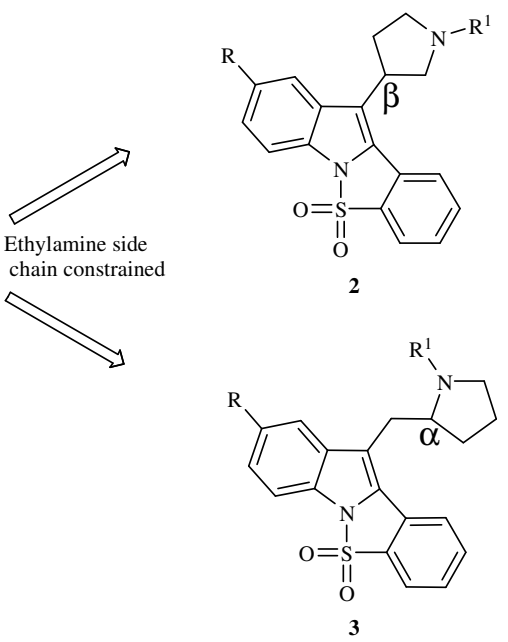

Fig. 1. Genesis of ligands $\mathbf{2}$ and $\mathbf{3}$

temperature and washed with diethyl ether. The aqueous phase was acidified using hydrochloric acid and the resulting solids were filtered, washed with water and dried. 3-Indolylsuccinic acid (2.6 g, $11.15 \mathrm{mmol})$, thus obtained, was reacted with 1,3dimethyl urea ( $3 \mathrm{~g}, 34.09 \mathrm{mmol})$ at $180^{\circ} \mathrm{C}$ and stirred for $1 \mathrm{~h}$. The hot mixture was diluted with water, cooled to room temperature and the resulting solids were filtered and further purified by flash chromatography (silica gel, EtOAc/hexanes, 2/3) to obtain 5a (1.7 g, $68 \%$ ), which was confirmed by IR, NMR and mass spectral analyses. Similarly compounds $\mathbf{5 b - 5 h}$ were synthesized.

5a: M.R $\left({ }^{\circ} \mathrm{C}\right)$ : 173.5-175.0; IR (KBr, $\left.v_{\max }, \mathrm{cm}^{-1}\right)$ : 3334, 1769, 1677, 745; mass $(\mathrm{m} / \mathrm{z}): 229[\mathrm{M}+1]$; ${ }^{1} \mathrm{H}$ NMR (400 $\mathrm{MHz})\left(\mathrm{CDCl}_{3}\right) \delta$ (ppm): 2.75-2.81 (1H, m), $2.91(3 \mathrm{H}, \mathrm{s}, \mathrm{N}-$ $\left.\mathrm{CH}_{3}\right), 3.15-3.25(1 \mathrm{H}, \mathrm{m}), 4.33-4.37(1 \mathrm{H}, \mathrm{m}), 7.10-7.27(2 \mathrm{H}$, m), 7.45-7.47 (1H, m), 8.02-8.07 (2H, m), 8.77 (1H, bs).

3-(1-Methyl pyrrolidin-3-yl)-1 $\boldsymbol{H}$-indole (6a): To a stirred suspension of lithium aluminum hydride $(0.85 \mathrm{~g}, 22.27 \mathrm{mmol})$ in tetrahydrofuran $(15 \mathrm{~mL})$ was added a solution of compound 5a $(1.7 \mathrm{~g}, 7.42 \mathrm{mmol})$ in tetrahydrofuran $(20 \mathrm{~mL})$ and the resulting slurry was stirred for $4 \mathrm{~h}$ at reflux temperature. After the completion of reaction, the reaction mixture was cooled to $10{ }^{\circ} \mathrm{C}$, poured on to chilled water $(20 \mathrm{~mL})$ and stirred for 0.5 h. The slurry, thus obtained, was filtered through celite pad and washed with ethyl acetate. The organic layer was separated and the aqueous phase was extracted with ethyl acetate $(2 \times 20 \mathrm{~mL})$. The combined organic layer was washed with brine solution $(15 \mathrm{~mL})$ and dried over sodium sulfate. Organic volatiles were removed under reduced pressure. The residual mass, thus obtained, was purified by flash chromatography (silica gel, EtOAc/TEA, 99/1) to afford compound $6 \mathbf{6}(0.52 \mathrm{~g}$, $70 \%$ ), which was confirmed by IR, NMR and mass spectral analyses. Similarly compounds $\mathbf{6 b}-\mathbf{6 h}$ were synthesized.

6a: M.R $\left({ }^{\circ} \mathrm{C}\right)$ : 85.3-89.6; IR (KBr, $\left.v_{\max } \mathrm{cm}^{-1}\right)$ : 3409, 2940, 1452, 737; mass $(\mathrm{m} / \mathrm{z}): 201[\mathrm{M}+1]$; ${ }^{1} \mathrm{H}$ NMR $(400 \mathrm{MHz})$ $\left(\mathrm{CDCl}_{3}\right) \delta(\mathrm{ppm}): 1.94-1.98(1 \mathrm{H}, \mathrm{m}), 2.37-2.40(1 \mathrm{H}, \mathrm{m}), 2.42$ $(3 \mathrm{H}, \mathrm{s}), 2.52-2.57(1 \mathrm{H}, \mathrm{m}), 2.60-2.65(1 \mathrm{H}, \mathrm{m}), 2.82-2.86(1 \mathrm{H}$, $\mathrm{m}), 3.10-3.15(1 \mathrm{H}, \mathrm{m}), 3.56-3.61(1 \mathrm{H}, \mathrm{m}), 7.10-7.13(1 \mathrm{H}, \mathrm{m})$, 7.21-7.25 (1H, m), 7.39-7.41 (1H, m), 7.68-7.72 (1H, d, $J=$ $8.04 \mathrm{~Hz}), 7.77-7.78(1 \mathrm{H}, \mathrm{d}, J=7.84 \mathrm{~Hz}), 8.32(1 \mathrm{H}, \mathrm{bs})$.

1-(2-Bromo benzenesulfonyl)-3-(1-methyl pyrrolidin3-yl)-1H-indole (7a ): 2-Bromobenzenesulfonyl chloride (0.76 $\mathrm{g}, 2.98 \mathrm{mmol})$ solution in dimethylformamide $(5 \mathrm{~mL})$ was added to a stirred solution of compound $\mathbf{6 a}(0.5 \mathrm{~g}, 2.48 \mathrm{mmol})$ and sodium hydride $(60 \%, 0.18 \mathrm{~g}, 3.73 \mathrm{mmol})$ in dimethyl formamide $(20 \mathrm{~mL})$ under nitrogen at room temperature. The reaction mass was stirred for $3 \mathrm{~h}$ at room temperature. After the completion of reaction, the reaction mixture was quenched with $25 \mathrm{~mL}$ ice-cold water and the product was extracted with ethyl acetate $(3 \times 15 \mathrm{~mL})$. The combined organic phase was washed sequentially with water and brine, dried over anhydrous sodium sulphate and concentrated in vacuo. The resultant residue was purified by flash chromatography (silica gel, EtOAc/TEA, 99/1) to afford the title compound 7a (0.83 g, 80 $\%$, which was confirmed by IR, NMR and mass analyses. Similarly compounds $\mathbf{7 b}-\mathbf{7 h}$ were synthesized.

7a: M.R $\left({ }^{\circ} \mathrm{C}\right)$ : 86.2-88.0; IR (KBr, $\left.v_{\max } \mathrm{cm}^{-1}\right): 2777,1369$, 1178, 593; Mass $(\mathrm{m} / \mathrm{z}): 419[\mathrm{M}+1], 421[\mathrm{M}+3]$; ${ }^{1} \mathrm{H}$ NMR $(400 \mathrm{MHz})\left(\mathrm{CDCl}_{3}\right) \delta(\mathrm{ppm}): 1.96-1.98(1 \mathrm{H}, \mathrm{m}), 2.36-2.40$ $(1 \mathrm{H}, \mathrm{m}), 2.43(3 \mathrm{H}, \mathrm{s}), 2.54-2.61(2 \mathrm{H}, \mathrm{m}), 2.83-2.87(1 \mathrm{H}, \mathrm{m})$, 3.10-3.15 (1H, m), 3.59-3.61 (1H, m), 7.22-7.24 (2H, m), 7.35$7.40(1 \mathrm{H}, \mathrm{m}), 7.43-7.48(1 \mathrm{H}, \mathrm{m}), 7.57-7.62(2 \mathrm{H}, \mathrm{m}), 7.65-$ $7.69(2 \mathrm{H}, \mathrm{m}), 8.02-8.04(1 \mathrm{H}, \mathrm{dd}, J=8.72$ and $2.16 \mathrm{~Hz})$.

10-(1-Methyl pyrrolidin-3-yl)-5-thia-4b-aza-indeno[2,1-a]indene-5,5-dioxide (2a): A solution of compound 7a (0.5 g, $1.19 \mathrm{mmol})$, potassium acetate $(0.23 \mathrm{~g}, 2.38 \mathrm{mmol})$ and tetrakis(triphenyl phosphine) palladium(0) (0.068 g, 0.059 mmole) in N,N-dimethyl acetamide $(10 \mathrm{~mL})$ was stirred for $5 \mathrm{~h}$ at $125-130^{\circ} \mathrm{C}$ under nitrogen atmosphere. After the completion of reaction, the resulting solution was partitioned between water and ethyl acetate. The layers were separated and the aqueous layer was extracted with ethyl acetate. The combined organic layer was washed with brine solution and dried over anhydrous sodium sulphate and the solvent was removed under reduced pressure. The residual mass, thus obtained, was purified by flash chromatography (silica gel, EtOAc/TEA, 99.5/0.5) to give 2a (0.31 g, $78 \%)$, which was confirmed by IR, NMR and mass spectral analyses. Similarly compounds $\mathbf{2 b - 2 h}$ were synthesized.

2a: M.R $\left({ }^{\circ} \mathrm{C}\right)$ : $167.8-173.1$; IR $\left(\mathrm{KBr}, \mathrm{v}_{\max } \mathrm{cm}^{-1}\right)$ : 2940, 1325, 1181, 583; Mass $(\mathrm{m} / \mathrm{z}): 339[\mathrm{M}+1]$; ${ }^{1} \mathrm{H}$ NMR (400 $\mathrm{MHz})\left(\mathrm{CDCl}_{3}\right) \delta(\mathrm{ppm}): 2.20-2.25(1 \mathrm{H}, \mathrm{m}), 2.33-2.37(1 \mathrm{H}$, m), $2.50(3 \mathrm{H}, \mathrm{s}), 2.83-2.97(4 \mathrm{H}, \mathrm{m}), 4.02-4.07(1 \mathrm{H}, \mathrm{m}), 7.24-$ 7.25 (1H, m), 7.35-7.40 (1H, m), 7.48-7.51 (1H, m), 7.64- 
$7.68(1 \mathrm{H}, \mathrm{m}), 7.70-7.72(1 \mathrm{H}, \mathrm{d}, J=7.72 \mathrm{~Hz}), 7.83-7.85(1 \mathrm{H}$, $\mathrm{d}, J=8.12 \mathrm{~Hz}), 7.87-7.89(1 \mathrm{H}, \mathrm{d}, J=8.12 \mathrm{~Hz}), 8.05-8.07$ $(1 \mathrm{H}, \mathrm{d}, J=8.00 \mathrm{~Hz})$.

2b: M.R ( $\left.{ }^{\circ} \mathrm{C}\right)$ : 97.7-106.8; IR ( $\left.\mathrm{KBr}, v_{\max } \mathrm{cm}^{-1}\right)$ : 2924, 1334, 1180, 582; Mass $(\mathrm{m} / \mathrm{z}): 353[\mathrm{M}+1]$; ${ }^{1} \mathrm{H}$ NMR $(400 \mathrm{MHz})$ $\left(\mathrm{CDCl}_{3}\right) \delta(\mathrm{ppm}): 1.14-1.17(3 \mathrm{H}, \mathrm{t}, J=7.24 \mathrm{~Hz}), 2.17-2.24$ $(1 \mathrm{H}, \mathrm{m}), 2.33-2.37(1 \mathrm{H}, \mathrm{m}), 2.59-2.75(3 \mathrm{H}, \mathrm{m}), 2.91-3.03(3 \mathrm{H}$, $\mathrm{m})$, 4.04-4.07 (1H, m), 7.23-7.29 (1H, m), 7.36-7.40 (1H, m), 7.46-7.50 $(1 \mathrm{H}, \mathrm{m}), 7.65-7.70(1 \mathrm{H}, \mathrm{m}), 7.71-7.73(1 \mathrm{H}, \mathrm{d}, J=$ $8.12 \mathrm{~Hz}), 7.83-7.86(2 \mathrm{H}, \mathrm{m}), 8.12-8.13(1 \mathrm{H}, \mathrm{d}, J=7.92 \mathrm{~Hz})$.

2c: M.R ( $\left.{ }^{\circ} \mathrm{C}\right)$ : 132.3-143.5; IR ( $\left.\mathrm{KBr}, \mathrm{v}_{\max } \mathrm{cm}^{-1}\right)$ : 2796, 1325, 1165, 580; Mass $(\mathrm{m} / \mathrm{z}): 369[\mathrm{M}+1]$; ${ }^{1} \mathrm{H}$ NMR $(400$ $\mathrm{MHz})\left(\mathrm{CDCl}_{3}\right) \delta(\mathrm{ppm}): 2.16-2.21(1 \mathrm{H}, \mathrm{m}), 2.32-2.35(1 \mathrm{H}$, m), 2.48 (3H, s), 2.75-2.77 (1H, m), 2.82-2.87 (1H, m), 2.93$2.97(2 \mathrm{H}, \mathrm{m}), 3.86(3 \mathrm{H}, \mathrm{s}), 3.97-4.01(1 \mathrm{H}, \mathrm{m}), 6.98-7.01(1 \mathrm{H}$, $\mathrm{dd}, J=8.80$ and $2.48 \mathrm{~Hz}), 7.44-7.48(2 \mathrm{H}, \mathrm{m}), 7.58-7.61(1 \mathrm{H}$, $\mathrm{d}, J=8.80 \mathrm{~Hz}), 7.63-7.67(1 \mathrm{H}, \mathrm{m}), 7.81-7.83(1 \mathrm{H}, \mathrm{d}, J=7.80$ $\mathrm{Hz}), 8.01-8.03(1 \mathrm{H}, \mathrm{d}, J=7.96 \mathrm{~Hz})$.

2d: M.R ( $\left.{ }^{\circ} \mathrm{C}\right)$ : 98.8-108.8; IR ( $\left.\mathrm{KBr}, v_{\max } \mathrm{cm}^{-1}\right): 2967,1332$, 1178, 580; Mass $(\mathrm{m} / \mathrm{z}): 383[\mathrm{M}+1]$; ${ }^{1} \mathrm{H}$ NMR $(400 \mathrm{MHz})$ $\left(\mathrm{CDCl}_{3}\right) \delta$ (ppm): 1.12-1.16 (3H, t, $\left.J=7.32 \mathrm{~Hz}\right), 2.16-2.21$ $(1 \mathrm{H}, \mathrm{m}), 2.35-2.37(1 \mathrm{H}, \mathrm{m}), 2.61-3.02(6 \mathrm{H}, \mathrm{m}), 3.87(3 \mathrm{H}, \mathrm{s})$, 4.03-4.05 (1H, m), 6.99-7.01 (1H, dd, $J=8.84$ \& 2.44 Hz), 7.44-7.48 (2H, m), 7.59-7.61 (1H, d, J=8.84 Hz), 7.63-7.66 $(1 \mathrm{H}, \mathrm{m}), 7.81-7.83(1 \mathrm{H}, \mathrm{d}, J=7.80 \mathrm{~Hz}), 8.06-8.08(1 \mathrm{H}, \mathrm{d}, J=$ $7.92 \mathrm{~Hz})$.

2e: M.R ( $\left.{ }^{\circ} \mathrm{C}\right)$ : 115.9-118.9; IR (KBr, $\left.v_{\max }, \mathrm{cm}^{-1}\right)$ : 2922, 1329, 1175, 559; mass $(\mathrm{m} / \mathrm{z}): 383[\mathrm{M}+1]$; ${ }^{1} \mathrm{H}$ NMR (400 $\mathrm{MHz})\left(\mathrm{CDCl}_{3}\right) \delta(\mathrm{ppm}): 1.42-1.47(3 \mathrm{H}, \mathrm{t}, J=6.96 \mathrm{~Hz}), 2.18-$ $2.22(1 \mathrm{H}, \mathrm{m}), 2.32-2.43(1 \mathrm{H}, \mathrm{m}), 2.49(3 \mathrm{H}, \mathrm{s}), 2.77-2.79(1 \mathrm{H}$, m), 2.86-2.88 (1H, m), 2.92-2.95 (2H, m), 3.94-3.99 (1H, m), 4.06-4.11 (2H, q, $J=6.96 \mathrm{~Hz}), 6.98-7.01(1 \mathrm{H}, \mathrm{dd}, J=8.80$ and $2.42 \mathrm{~Hz}), 7.44-7.48(2 \mathrm{H}, \mathrm{m}), 7.57-7.60(1 \mathrm{H}, \mathrm{d}, J=8.80$ $\mathrm{Hz}), 7.62-7.66$ (1H, m), 7.80-7.82 (1H, d, $J=7.84 \mathrm{~Hz}), 8.01-$ $8.03(1 \mathrm{H}, \mathrm{d}, J=7.92 \mathrm{~Hz})$.

2f: M.R $\left({ }^{\circ} \mathrm{C}\right)$ : $110-115.1 ; \mathrm{IR}\left(\mathrm{KBr}, \nu_{\max }, \mathrm{cm}^{-1}\right)$ : 2966, 1328, 1179, 559; mass $(\mathrm{m} / \mathrm{z}): 397[\mathrm{M}+1]$; ${ }^{1} \mathrm{H}$ NMR $(400 \mathrm{MHz})$ $\left(\mathrm{CDCl}_{3}\right) \delta$ (ppm): 1.17-1.21 (3H, t, $\left.J=7.20 \mathrm{~Hz}\right), 1.44-1.47$ $(3 \mathrm{H}, \mathrm{t}, J=6.96 \mathrm{~Hz}), 2.18-2.23(1 \mathrm{H}, \mathrm{m}), 2.32-2.38(1 \mathrm{H}, \mathrm{m})$, 2.59-2.68 (2H, m), 2.74-2.80 (1H, m), 2.88-2.90 (1H, m), 2.95$2.99(2 \mathrm{H}, \mathrm{m}), 3.98-4.00(1 \mathrm{H}, \mathrm{m}), 4.06-4.11(2 \mathrm{H}, \mathrm{q}, J=6.96$ $\mathrm{Hz}), 6.98-6.99(1 \mathrm{H}, \mathrm{dd}, J=8.80 \& 2.36 \mathrm{~Hz}), 7.44-7.48(2 \mathrm{H}$, m), 7.57-7.60 (1H, d, $J=8.80 \mathrm{~Hz}), 7.62-7.64(1 \mathrm{H}, \mathrm{m}), 7.80$ $7.82(1 \mathrm{H}, \mathrm{d}, J=7.80 \mathrm{~Hz}), 8.05-8.07(1 \mathrm{H}, \mathrm{d}, J=7.92 \mathrm{~Hz})$.

2g: M.R $\left({ }^{\circ} \mathrm{C}\right)$ : $141.8-145.7$; IR $\left(\mathrm{KBr}, v_{\max }, \mathrm{cm}^{-1}\right)$ : 2972, 1325, 1180, 586; mass $(\mathrm{m} / \mathrm{z}): 397[\mathrm{M}+1]$; ${ }^{1} \mathrm{H}$ NMR $(400$ $\mathrm{MHz})\left(\mathrm{CDCl}_{3}\right) \delta(\mathrm{ppm}): 1.36-1.38(6 \mathrm{H}, \mathrm{d}, J=6.40 \mathrm{~Hz}), 2.17-$ $2.20(1 \mathrm{H}, \mathrm{m}), 2.33(1 \mathrm{H}, \mathrm{m}), 2.48(3 \mathrm{H}, \mathrm{s}), 2.74-2.76(1 \mathrm{H}, \mathrm{m})$, 2.81-2.83 (1H, m), 2.90-2.94 (2H, m), 3.95-3.97 (1H, m), 4.53$4.56(1 \mathrm{H}, \mathrm{m}), 6.97-6.99(1 \mathrm{H}, \mathrm{dd}, J=8.80$ and $2.40 \mathrm{~Hz}), 7.46-$ $7.49(2 \mathrm{H}, \mathrm{m}), 7.57-7.59(1 \mathrm{H}, \mathrm{d}, J=8.80 \mathrm{~Hz}), 7.62-7.64$ $(1 \mathrm{H}, \mathrm{m}), 7.81-7.82(1 \mathrm{H}, \mathrm{d}, J=7.80 \mathrm{~Hz}), 8.00-8.01(1 \mathrm{H}, \mathrm{d}, J=$ $7.96 \mathrm{~Hz})$.

2h: IR (KBr, $\left.v_{\max }, \mathrm{cm}^{-1}\right): 2970,1335,1182,580 ;$ mass $(\mathrm{m} /$ z): $411[\mathrm{M}+1]$; ${ }^{1} \mathrm{H}$ NMR $(400 \mathrm{MHz})\left(\mathrm{CDCl}_{3}\right) \delta$ (ppm): $1.19-$ $1.25(3 \mathrm{H}, \mathrm{t}, J=7.24 \mathrm{~Hz}), 1.37-1.39(6 \mathrm{H}, \mathrm{d}, J=6.42 \mathrm{~Hz}), 2.19$ $2.25(1 \mathrm{H}, \mathrm{m}), 2.32-2.39(1 \mathrm{H}, \mathrm{m}), 2.59-2.76(3 \mathrm{H}, \mathrm{m}), 2.84-$ $2.86(1 \mathrm{H}, \mathrm{m}), 2.92-2.95(2 \mathrm{H}, \mathrm{m}), 4.01-4.06(1 \mathrm{H}, \mathrm{m}), 4.45-$ $4.48(1 \mathrm{H}, \mathrm{m}), 6.98-7.00(1 \mathrm{H}, \mathrm{dd}, J=8.84 \& 2.48 \mathrm{~Hz}), 7.44-$
$7.48(2 \mathrm{H}, \mathrm{m}), 7.55-7.59(1 \mathrm{H}, \mathrm{d}, J=8.84 \mathrm{~Hz}), 7.63-7.65(1 \mathrm{H}$, m), 7.80-7.81 (1H, d, $J=7.82 \mathrm{~Hz}), 7.99-8.00(1 \mathrm{H}, \mathrm{d}, J=7.90$ $\mathrm{Hz})$.

3-Bromo-1-(tert-butyl dimethyl silanyl)-1H-indole (8a): To a cooled solution of indole (4a, $2 \mathrm{~g}, 17.09 \mathrm{mmol})$ in tetrahydrofuran $(20 \mathrm{~mL})$ at $-78{ }^{\circ} \mathrm{C}$ was added a solution of n-butyllithium (32 mL, 1.6 M solution in hexane, $51.28 \mathrm{mmol}$ ) dropwise via cannula under nitrogen atmosphere. The reaction mass was warmed to $-10^{\circ} \mathrm{C}$, stirred for $0.5 \mathrm{~h}$ and again cooled to $-50{ }^{\circ} \mathrm{C}$. Then a solution of tert-butyl dimethyl silyl chloride (7.7 g, $51.28 \mathrm{mmol})$ in tetrahydrofuran $(30 \mathrm{~mL})$ was added dropwise to this mixture. The temperature was raised to $0{ }^{\circ} \mathrm{C}$ and stirred for $3 \mathrm{~h}$ at the same temperature. The reaction mixture was cooled to $-78^{\circ} \mathrm{C}$, freshly crystallized $N$-bromosuccinimide (6.06 g, $34.18 \mathrm{mmol}$ ) was added via a solid-addition funnel and the resulting mixture was stirred for $2 \mathrm{~h}$ at $-78^{\circ} \mathrm{C}$ and then allowed warm to room temperature. After completion of the reaction, the mass was poured into a mixture of hexane $(100 \mathrm{~mL})$ and pyridine $(1 \mathrm{~mL})$. The resulting suspension was filtered through a celite pad, the filtrate was concentrated under reduced pressure. The crude residue was then purified by flash chromatography (silica gel, $100 \%$ hexane) to obtain 8a $(3.4 \mathrm{~g}, 68 \%)$ as a colourless solid, which was confirmed by IR, NMR and mass analyses. Similarly compounds $\mathbf{8 b - 8 d}$ were synthesized.

8a: M.R $\left({ }^{\circ} \mathrm{C}\right)$ : 73.2-74.1; IR $\left(\mathrm{KBr}, \nu_{\mathrm{nax}}, \mathrm{cm}^{-1}\right): 2931,1445$, 1287, 739; Mass $(\mathrm{m} / \mathrm{z}): 310.2[\mathrm{M}+1], 312.2[\mathrm{M}+3] ;{ }^{1} \mathrm{H}$ NMR (400 MHz) $\left(\mathrm{CDCl}_{3}\right) \delta(\mathrm{ppm}): 0.60(6 \mathrm{H}, \mathrm{s}), 0.91(9 \mathrm{H}, \mathrm{s})$, 7.16-7.23 (3H, m), 7.47-7.50 (1H, m), 7.55-7.58 (1H, m).

(1H-Indol-3-yl)-(1-methyl pyrrolidin-2-yl)methanone (9a): To a stirred solution of compound $\mathbf{8 a}$ (1 g, $3.41 \mathrm{mmol})$ in tetrahydrofuran $(15 \mathrm{~mL})$ cooled to $-78^{\circ} \mathrm{C}$ was added a solution of $n$-butyllithium ( $6.4 \mathrm{~mL}, 1.6 \mathrm{M}$ solution in hexane, 10.23 $\mathrm{mmol}$ ) dropwise via cannula under nitrogen atmosphere. The resulting solution was stirred for $15 \mathrm{~min}$ at $-78^{\circ} \mathrm{C}$, then added a solution of 1-methyl pyrrolidine-2-carboxylate ( $0.65 \mathrm{~g}, 5.10$ mmol) in tetrahydrofuran $(10 \mathrm{~mL})$. Reaction mass was stirred for $12 \mathrm{~h}$ at room temperature. After completion of the reaction, the reaction mass was quenched in saturated ammonium chloride solution and the compound was extracted with ethyl acetate. The organic layer was washed with brine solution, dried over anhydrous sodium sulphate and solvent removed under reduced pressure. The residual mass, thus obtained, was purified by flash chromatography (silica gel, EtOAc/TEA, 99/1) to afford the compound 9a $(0.46 \mathrm{~g}, 60 \%)$, which was confirmed by IR, NMR and mass analyses. Similarly compounds 9b-9d were synthesized.

9a: IR (KBr, $\left.v_{\max }, \mathrm{cm}^{-1}\right)$ : 3314, 1769, 1277, 735; mass $(\mathrm{m} /$ $z): 229.3[\mathrm{M}+1] ;{ }^{1} \mathrm{H}$ NMR $(400 \mathrm{MHz})\left(\mathrm{CDCl}_{3}\right) \delta(\mathrm{ppm})$ : 1.85-1.90 (1H, m), 1.99-2.04 (2H, m), 2.25-2.28 (1H, m), 2.35$2.38(1 \mathrm{H}, \mathrm{m}), 2.40\left(3 \mathrm{H}, \mathrm{s}, \mathrm{N}-\mathrm{CH}_{3}\right), 3.22-3.26(1 \mathrm{H}, \mathrm{m}), 3.43-$ $3.47(1 \mathrm{H}, \mathrm{m}), 7.27-7.32(2 \mathrm{H}, \mathrm{m}), 7.42-7.44(1 \mathrm{H}, \mathrm{m}), 8.37-$ $8.38(1 \mathrm{H}, \mathrm{m}), 8.47-8.49(1 \mathrm{H}, \mathrm{m}), 8.84(1 \mathrm{H}, \mathrm{bs})$.

3-(1-Methyl pyrrolidin-2-ylmethyl)- $1 H$-indole (10a): To a stirred suspension of lithium aluminum hydride $(0.11 \mathrm{~g}$, $2.96 \mathrm{mmol})$ in tetrahydrofuran $(10 \mathrm{~mL})$ was added a solution of compound 9a $(0.45 \mathrm{~g}, 1.97 \mathrm{mmol})$ in tetrahydrofuran (10 $\mathrm{mL}$ ) and the resulting slurry was heated at reflux for $4 \mathrm{~h}$. After the completion of reaction, the reaction mixture was cooled to 
$10^{\circ} \mathrm{C}$, poured on to chilled water $(20 \mathrm{~mL})$ and stirred for $0.5 \mathrm{~h}$. The slurry, thus obtained, was filtered through celite pad and washed with ethyl acetate $(15 \mathrm{~mL})$. The organic layer was separated and the aqueous phase was extracted with ethyl acetate $(2 \times 15 \mathrm{~mL})$. The combined organic layer was washed with brine solution $(15 \mathrm{~mL})$ and dried over anhydrous sodium sulfate. Organic volatiles were removed under reduced pressure. The residual mass, thus obtained, was purified by flash chromatography (silica gel, EtOAc/TEA, 99/1) to afford compound 10a (0.35 g, $83 \%)$, which was confirmed by IR, NMR and mass analyses. Similarly compounds 10b-10d were synthesized.

10a: IR (KBr, $\left.v_{\max }, \mathrm{cm}^{-1}\right): 3399,2920,1472,717$; mass $(\mathrm{m} / \mathrm{z}): 215.2[\mathrm{M}+1] ;{ }^{1} \mathrm{H} \mathrm{NMR}(400 \mathrm{MHz})\left(\mathrm{CDCl}_{3}\right) \delta(\mathrm{ppm})$ : 1.58-1.61 (2H, m), 1.77-1.80 (2H, m), 2.21-2.23 (1H, m), 2.46$2.50(4 \mathrm{H}, \mathrm{m}), 2.58-2.64(1 \mathrm{H}, \mathrm{m}), 3.13-3.22(2 \mathrm{H}, \mathrm{m}), 7.02-$ $7.03(1 \mathrm{H}, \mathrm{m}), 7.11-7.14(1 \mathrm{H}, \mathrm{m}), 7.17-7.21(1 \mathrm{H}, \mathrm{m}), 7.35-$ 7.37 (1H, m), 7.60-7.62 (1H, d, $J=7.84 \mathrm{~Hz}), 8.00$ (1H, bs).

1-(2-Bromo benzenesulfonyl)-3-(1-methyl pyrrolidin2-ylmethyl)-1H-indole (11a): 2-Bromobenzenesulfonyl chloride $(0.50 \mathrm{~g}, 1.96 \mathrm{mmol})$ in dimethylformamide $(5 \mathrm{~mL})$ was added to a stirred solution of compound 10a $(0.35 \mathrm{~g}, 1.63$ $\mathrm{mmol}$ ) and sodium hydride (50\%, $0.098 \mathrm{~g}, 2.45 \mathrm{mmol})$ in dimethylformamide $(15 \mathrm{~mL})$ under nitrogen atmosphere at room temperature and the reaction mass was stirred for $3 \mathrm{~h}$ at room temperature. After the completion of reaction, the reaction mixture was quenched with $15 \mathrm{~mL}$ ice-cold water and the product was extracted with ethyl acetate $(3 \times 15 \mathrm{~mL})$. The combined organic phase was washed sequentially with water and brine, dried over anhydrous sodium sulphate and concentrated in vacuo. The resultant residue was purified by flash chromatography ( silica gel, EtOAc/TEA, 99/1) to afford the title compound 11a $(0.56 \mathrm{~g}, 80 \%)$, which was confirmed by IR, NMR and mass analyses. Similarly compounds $\mathbf{1 1 b}$ 11d were synthesized.

11a: M.R $\left({ }^{\circ} \mathrm{C}\right): 86.2-88.0 ; \mathrm{IR}\left(\mathrm{KBr}, \mathrm{v}_{\mathrm{nax}}, \mathrm{cm}^{-1}\right): 2765,1369$, 1178, 596; mass $(\mathrm{m} / \mathrm{z}): 433[\mathrm{M}+1], 435[\mathrm{M}+3]$; ${ }^{1} \mathrm{H}$ NMR $(400 \mathrm{MHz})\left(\mathrm{CDCl}_{3}\right) \delta(\mathrm{ppm}): 1.54-1.58(2 \mathrm{H}, \mathrm{m}), 1.64-1.68$ $(2 \mathrm{H}, \mathrm{m}), 2.22-2.24(1 \mathrm{H}, \mathrm{m}), 2.48-2.53(4 \mathrm{H}, \mathrm{m}), 2.56-2.59(1 \mathrm{H}$, $\mathrm{m}), 3.10-3.14(2 \mathrm{H}, \mathrm{m}), 7.21-7.25(2 \mathrm{H}, \mathrm{m}), 7.36-7.41(1 \mathrm{H}, \mathrm{m})$, 7.45-7.49 (1H, m), 7.52-7.57 (1H, m), $7.58(1 \mathrm{H}, \mathrm{s}), 7.65-7.77$ $(2 \mathrm{H}, \mathrm{m}), 8.04-8.07(1 \mathrm{H}, \mathrm{dd}, J=7.88$ and $1.68 \mathrm{~Hz})$.

10-(1-Methyl pyrrolidin-2-ylmethyl)-5-thia-4b-aza indeno[2,1-a]indene-5,5-dioxide (3a): A solution of compound $11 \mathrm{a}(0.55 \mathrm{~g}, 1.27 \mathrm{mmol})$, potassium acetate $(0.25 \mathrm{~g}, 2.55 \mathrm{mmol})$ and tetrakis(triphenyl phosphine) palladium(0) $(0.073 \mathrm{~g}, 0.063$ $\mathrm{mmol})$ in N,N-dimethyl acetamide $(10 \mathrm{~mL})$ was stirred for $5 \mathrm{~h}$ at $125-130{ }^{\circ} \mathrm{C}$ under nitrogen atmosphere. After the completion of reaction, the resulting solution was partitioned between water and ethyl acetate. The organic layer was separated and the aqueous phase was extracted with ethyl acetate. The combined organic layer was washed with brine solution and dried over anhydrous sodium sulphate and the solvent was removed under reduced pressure. The residual mass, thus obtained was purified by flash chromatography (silica gel, EtOAc/TEA, $99.5 / 0.5)$ to give $3 \mathbf{a}(0.35 \mathrm{~g}, 80 \%)$, which was confirmed by IR, NMR and mass analyses. Similarly compounds $\mathbf{3 b - 3 d}$ were synthesized. 3a: M.R $\left({ }^{\circ} \mathrm{C}\right)$ : 200.6-201.9; IR $\left(\mathrm{KBr}, \mathrm{v}_{\operatorname{nax}}, \mathrm{cm}^{-1}\right): 2788$, 1332, 1177, 547; mass $(\mathrm{m} / \mathrm{z}): 353[\mathrm{M}+1]$; ${ }^{1} \mathrm{H}$ NMR (400 $\mathrm{MHz})\left(\mathrm{CDCl}_{3}\right) \delta(\mathrm{ppm}): 1.60-1.80(4 \mathrm{H}, \mathrm{m}), 2.25-2.27(1 \mathrm{H}$, m), 2.54-2.62 (4H, m), 2.87-2.93 (1H, m), 3.14-3.20 (1H, m), 3.32-3.35 (1H, m), 7.25-7.28 (1H, m), 7.36-7.38 (1H, m), 7.48$7.50(1 \mathrm{H}, \mathrm{m}), 7.59-7.61(1 \mathrm{H}, \mathrm{d}, J=8.00 \mathrm{~Hz}), 7.64-7.68(1 \mathrm{H}$, $\mathrm{m}), 7.69-7.71(1 \mathrm{H}, \mathrm{d}, J=8.00 \mathrm{~Hz}), 7.83-7.85(1 \mathrm{H}, \mathrm{d}, J=7.82$ $\mathrm{Hz}), 7.88-7.90(1 \mathrm{H}, \mathrm{d}, J=7.84 \mathrm{~Hz})$.

3b: M.R ( $\left.{ }^{\circ} \mathrm{C}\right)$ : 190.7-195.1; IR (KBr, $\left.v_{\text {nax }}, \mathrm{cm}^{-1}\right): 2769$, 1322, 1174, 582; mass $(\mathrm{m} / \mathrm{z}): 383[\mathrm{M}+1]$; ${ }^{1} \mathrm{H}$ NMR $(400$ $\mathrm{MHz})\left(\mathrm{CDCl}_{3}\right) \delta(\mathrm{ppm}): 1.62-1.65(2 \mathrm{H}, \mathrm{m}) .1 .78-1.80(2 \mathrm{H}$, m), 2.27-2.29 (1H, m), 2.55-2.63 (4H, m), 2.86-2.90 (1H, m), 3.14-3.19 (1H, m), 3.28-3.32 (1H, m), 3.86 (3H, s), 6.99-7.01 $(1 \mathrm{H}, \mathrm{dd}, J=8.84$ and $2.00 \mathrm{~Hz}), 7.02-7.05(1 \mathrm{H}, \mathrm{m}), 7.45-7.49$ $(1 \mathrm{H}, \mathrm{m}), 7.58-7.60(1 \mathrm{H}, \mathrm{d}, J=8.84 \mathrm{~Hz}), 7.63-7.66(1 \mathrm{H}, \mathrm{m})$, $7.81-7.83(1 \mathrm{H}, \mathrm{d}, J=7.83 \mathrm{~Hz}), 7.81-7.88(1 \mathrm{H}, \mathrm{d}, J=7.84$ $\mathrm{Hz})$.

3c: M.R $\left({ }^{\circ} \mathrm{C}\right)$ : 113.0-120.0; IR (KBr, $\left.v_{\text {nax }}, \mathrm{cm}^{-1}\right)$ : 2774 , 1334, 1174, 584; mass $(\mathrm{m} / \mathrm{z}): 397$ [M+1]; ${ }^{1} \mathrm{H}$ NMR (400 MHz) $\left(\mathrm{CDCl}_{3}\right) \delta(\mathrm{ppm}): 1.43-1.46(3 \mathrm{H}, \mathrm{t}, J=6.96 \mathrm{~Hz}), 1.60-1.64$ (2H, m). 1.77-1.81 (2H, m), 2.26-2.28 (1H, m), 2.54-2.61 (4H, $\mathrm{m}), 2.84-2.90(1 \mathrm{H}, \mathrm{m}), 3.13-3.18(1 \mathrm{H}, \mathrm{m}), 3.27-3.31(1 \mathrm{H}, \mathrm{m})$, 4.05-4.10 (2H, q, $J=6.96 \mathrm{~Hz}), 6.98-7.01(1 \mathrm{H}, \mathrm{dd}, J=8.71$ and $2.20 \mathrm{~Hz}), 7.02-7.05(1 \mathrm{H}, \mathrm{m}), 7.44-7.48(1 \mathrm{H}, \mathrm{m}), 7.57-$ $7.59(1 \mathrm{H}, \mathrm{d}, J=8.71 \mathrm{~Hz}), 7.62-7.66(1 \mathrm{H}, \mathrm{m}), 7.81-7.83(1 \mathrm{H}$, $\mathrm{d}, J=7.82 \mathrm{~Hz}), 7.85-7.87(1 \mathrm{H}, \mathrm{d}, J=7.86 \mathrm{~Hz})$.

3d: M.R ( $\left.{ }^{\circ} \mathrm{C}\right)$ : 123.6-125.1; IR (KBr, $\left.v_{\max }, \mathrm{cm}^{-1}\right)$ : 2777 , 1326, 1174, 580; mass $(\mathrm{m} / \mathrm{z}): 411[\mathrm{M}+1]$; ${ }^{1} \mathrm{H}$ NMR (400 $\mathrm{MHz})\left(\mathrm{CDCl}_{3}\right) \delta(\mathrm{ppm}): 1.35-1.37(6 \mathrm{H}, \mathrm{d}, J=6.40 \mathrm{~Hz}), 1.60-$ $1.64(2 \mathrm{H}, \mathrm{m}) .1 .77-1.81(2 \mathrm{H}, \mathrm{m}), 2.26-2.28(1 \mathrm{H}, \mathrm{m}), 2.55-$ $2.62(4 \mathrm{H}, \mathrm{m}), 2.84-2.90(1 \mathrm{H}, \mathrm{m}), 3.12-3.18(1 \mathrm{H}, \mathrm{m}), 3.26-$ $3.31(1 \mathrm{H}, \mathrm{m}), 4.53-4.56(1 \mathrm{H}, \mathrm{m}), 6.98-7.00(1 \mathrm{H}, \mathrm{dd}, J=8.80$ and $2.0 \mathrm{~Hz}), 7.01-7.04(1 \mathrm{H}, \mathrm{m}), 7.44-7.48(1 \mathrm{H}, \mathrm{m}), 7.56-7.58$ $(1 \mathrm{H}, \mathrm{d}, J=8.80 \mathrm{~Hz}), 7.62-7.66(1 \mathrm{H}, \mathrm{m}), 7.80-7.87(1 \mathrm{H}, \mathrm{d}, J=$ $7.80 \mathrm{~Hz}), 7.85-7.87(1 \mathrm{H}, \mathrm{d}, J=7.80 \mathrm{~Hz})$.

\section{RESULTS AND DISCUSSION}

The target compounds were synthesized according to the representative Schemes I and II.

Indole-3-substituted pyrrolidine diones (5a-5h) were synthesized by reacting equimolar amounts of indole and maleic acid at $50{ }^{\circ} \mathrm{C}$. The resulting indole-3-succinic acid derivatives were further reacted with dialkyl ureas at $180{ }^{\circ} \mathrm{C}$. The diones, thus obtained, were reduced using lithium aluminum hydride to the corresponding pyrrolidine compounds (6a-6h). The intermediates (6a-6h) were reacted with 2-bromobenzenesulfonyl chloride in the presence of a base to obtain the intermediates (7a-7h), which were further subjected to Heck cyclization reaction to obtain the desired series of compounds (2a-2h).

N-Silylated-3-bromo indole derivatives (8a-8d) were synthesized by protection of indoles with substituted silyl chlorides in the presence of $n$-butyllithium, followed by bromination at $\mathrm{C}-3$ of indole position using $\mathrm{N}$-bromo succinimide. The intermediates $\mathbf{8 a - 8 d}$ were reacted with $\mathrm{N}$-methyl proline esters in the presence of $n$-butyl lithium resulting in indole-3methanone derivatives (9a-9d). These methanone derivatives were reduced by using lithium aluminum hydride to obtain 


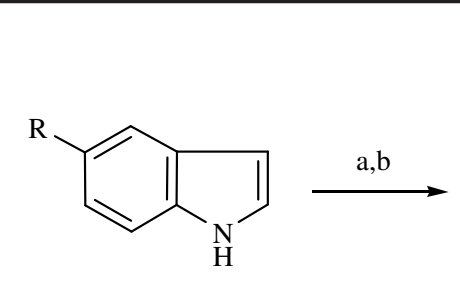

(4a-d)<smiles>[R]c1ccc2[nH]cc(C3CC(=O)N([R])C3=O)c2c1</smiles>

$(\mathbf{5 a}-\mathbf{h})$<smiles>[R]c1ccc2[nH]cc(C3CCN([R])C3)c2c1</smiles>

(6a-h)<smiles>[R]/[R](=[X])=C/COCC</smiles><smiles></smiles>

(2a-h)

Scheme-I: (a) Maleic acid, $50{ }^{\circ} \mathrm{C}, 1 \mathrm{~h}$; (b) 1,3-Dialkylurea, $180-190{ }^{\circ} \mathrm{C}, 1 \mathrm{~h}, 68 \%$; (c) LAH, THF, reflux, $4 \mathrm{~h}, 70 \%$; (d) 2-Bromo benzenesulfonyl chloride, $\mathrm{NaH}, \mathrm{DMF}, \mathrm{RT}, 3 \mathrm{~h}, 80 \%$; (e) $\mathrm{CH}_{3} \mathrm{COOK}, \mathrm{Pd}\left(\mathrm{PPh}_{3}\right)_{4}$, DMA, $130{ }^{\circ} \mathrm{C}, 5 \mathrm{~h}, 78 \%$<smiles>[R]c1ccc2[nH]ccc2c1</smiles>

(4a-d)<smiles>[R]c1ccc2c(c1)c(Br)cn2[Si](C)(C)C(C)(C)C</smiles><smiles>C1CCCCC1</smiles><smiles>[R]c1ccc2c(c1)c(CC1CCCN1[R])cn2S(=O)(=O)c1ccccc1Br</smiles>

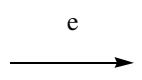

$\mathrm{R}=\mathrm{H}, \mathrm{OC}$
$\mathrm{R}^{1}=\mathrm{CH}_{3}$<smiles>[R]c1ccc2[nH]cc(C(=O)C3CCCN3[R])c2c1</smiles>

(9a-d)<smiles>[R]c1ccc2[nH]cc(CC3CCCN3[R])c2c1</smiles>

(10a-d) (a) $n$-BuLi, tert-butyl dimethyl silyl chloride, NBS, THF, -78 ${ }^{\circ} \mathrm{C}, 6 \mathrm{~h}, 68 \%$; (b) $n$-BuLi, 1 -alkyl pyrrolidine-2-carboxylic acid methyl ester,
THF, $-78^{\circ} \mathrm{C}, 12 \mathrm{~h}, 60 \%$; (c) LAH, THF, reflux, 4 h, $83 \%$; (d) 2-Bromo benzenesulfonyl chloride, NaH, DMF, RT, 3 h, $80 \%$; (e) $\mathrm{CH}_{3} \mathrm{COOK}$, $\mathrm{Pd}\left(\mathrm{PPh}_{3}\right)_{4}, \mathrm{DMA}, 130^{\circ} \mathrm{C}, 5 \mathrm{~h}, 80 \%$

corresponding amines $(\mathbf{1 0 a}-\mathbf{1 0 d})$. The intermediates $(\mathbf{1 0 a}-\mathbf{1 0 d})$ were reacted with 2-bromo benzenesulfonyl chloride in the presence of base to afford the intermediates (11a-11d), which were further subjected to the familiar Heck reaction to obtain the target compounds (3a-3d). All the intermediates and final compounds were fully characterized by IR, NMR and mass spectral data.

Structure activity relationship (SAR): Analytically well characterized 10-(1-methyl pyrrolidin-3-yl)-5-thia-4b-azaindeno[2,1-a]indene-5,5-dioxide (2a-2h) and 10-(1-methyl pyrrolidin-2-ylmethyl)-5-thia-4b-aza-indeno[2,1-a]indene5,5-dioxide (3a-3d) derivatives were tested for their 5- $\mathrm{HT}_{6} \mathrm{R}$ in vitro binding affinity at $100 \mathrm{nM}$ concentration. All the compounds were tested as racemates and the results are given in Table-1. As part of our structure activity relationship studies, initially we regidized the tryptamine side chain into the pyrrolidine ring through $\beta$ carbon i.e., 10-(1-methyl pyrrolidin3-yl)-5-thia-4b-aza-indeno[2,1-a]indene-5,5-dioxide (unsubstituted indole 2a) and the $\%$ inhibition at $100 \mathrm{nM}$ concentration was found to be 54.65 percent. Later, we introduced 
TABLE-2

PHARMACOKINETIC PROFILE OF COMPOUND 2 $\mathrm{c}^{\mathrm{a}}$

\begin{tabular}{cccccccc}
\hline Route & $\mathrm{n}$ & Dose $(\mathrm{mg} / \mathrm{kg})$ & $\mathrm{C}_{\max }(\mathrm{ng} / \mathrm{mL})$ & $\mathrm{T}_{\max }(\mathrm{h})$ & $\mathrm{AUC}_{\mathrm{t}}(\mathrm{ng} \mathrm{h} / \mathrm{mL})$ & $\mathrm{t}_{1 / 2}(\mathrm{~h})$ & $\mathrm{F}(\%)$ \\
\hline Oral & 3 & 10 & $107 \pm 49$ & $1.3 \pm 0.6$ & $946 \pm 563$ & $7.48 \pm 0.85$ & 34 \\
i.v. & 3 & 10 & $1476 \pm 455$ & $0.08 \pm 0.0$ & $2769 \pm 307$ & $4.13 \pm 0.31$ & \\
\hline
\end{tabular}

${ }^{a}$ Fasted male wistar rats, Vehicle used: water for injection for both oral and i.v. routes. Dosing Volumes: $10 \mathrm{~mL} / \mathrm{kg}$ for oral and $2 \mathrm{~mL} / \mathrm{kg}$ for $i . v$.

the substitution on phenyl ring of indole with electron donating groups like methoxy (2c, $95.52 \%)$, ethoxy (2e, $58.54 \%)$, isopropoxy (2g, $53 \%)$ at second position $(\mathbf{R})$ and these were found to have moderate affinities at $5-\mathrm{HT}_{6} \mathrm{R}$ and it appears that there is a slight preference for lower alkoxy (methoxy) substitution. Replacement of methyl with ethyl on the amine had little effect on $5-\mathrm{HT}_{6} \mathrm{R}$ binding affinities as can be seen by comparing 2c (95.52\%) with $\mathbf{2 d}(66.63 \%)$ and $\mathbf{2 e}(58.54 \%)$ with 2 f $(41.97 \%)$. Based on these results, we evaluated the effect of rigidization of the tryptamine side chain into the pyrrolidine ring through $\alpha$ carbon. This change resulted in compounds with less potency compared to their respective counterparts as can be seen by comparing $\mathbf{2 c}(95.52 \%)$ vs $\mathbf{3 b}$ $(63.42 \%)$ and 2 e $(58.54 \%)$ vs 3 c $(52.36 \%))$. The overall result indicates that rigidization through $\alpha$ and $\beta$ carbons are tolerated for $5-\mathrm{HT}_{6} \mathrm{R}$ binding. Among all the synthesized compounds, $2 \mathbf{c}(95.52 \%)$ has shown highest percent of inhibition at $5-\mathrm{HT}_{6} \mathrm{R}$, when tested at $100 \mathrm{nM}$ concentration, indicating that regidization of the tryptamine side chain into the pyrrolidine ring through $\beta$ carbon was more favorable than rigidization through $\alpha$ carbon.

\begin{tabular}{|c|c|c|c|}
\hline \multicolumn{4}{|c|}{$\begin{array}{c}\text { TABLE-1 } \\
\text { 5-HT }{ }_{6} \text { R BINDING AFFINITIES OF } \\
\text { COMPOUND } 2 \text { AND } 3 \text { DERIVATIVES }^{\mathrm{a}}\end{array}$} \\
\hline Compound & $\mathrm{R}$ & $\overline{R^{1}}$ & Inhibition \% at $100 \mathrm{nM}$ \\
\hline $2 a$ & $\mathrm{H}$ & $\mathrm{CH}_{3}$ & 54.65 \\
\hline $2 b$ & $\mathrm{H}$ & $\mathrm{C}_{2} \mathrm{H}_{5}$ & 43.76 \\
\hline $2 c$ & $\mathrm{OCH}_{3}$ & $\mathrm{CH}_{3}$ & 95.52 \\
\hline 2d & $\mathrm{OCH}_{3}$ & $\mathrm{C}_{2} \mathrm{H}_{5}$ & 66.63 \\
\hline $2 e$ & $\mathrm{OC}_{2} \mathrm{H}_{5}$ & $\mathrm{CH}_{3}$ & 58.54 \\
\hline $2 f$ & $\mathrm{OC}_{2} \mathrm{H}_{5}$ & $\mathrm{C}_{2} \mathrm{H}_{5}$ & 41.97 \\
\hline $2 \mathrm{~g}$ & $\mathrm{OCH}\left(\mathrm{CH}_{3}\right)_{2}$ & $\mathrm{CH}_{3}$ & 53.00 \\
\hline $2 \mathrm{~h}$ & $\mathrm{OCH}\left(\mathrm{CH}_{3}\right)_{2}$ & $\mathrm{C}_{2} \mathrm{H}_{5}$ & 31.93 \\
\hline $\mathbf{3 a}$ & $\mathrm{H}$ & $\mathrm{CH}_{3}$ & 58.61 \\
\hline $\mathbf{3 b}$ & $\mathrm{OCH}_{3}$ & $\mathrm{CH}_{3}$ & 63.42 \\
\hline $3 c$ & $\mathrm{OC}_{2} \mathrm{H}_{5}$ & $\mathrm{CH}_{3}$ & 52.36 \\
\hline 3d & $\mathrm{OCH}\left(\mathrm{CH}_{3}\right)_{2}$ & $\mathrm{CH}_{3}$ & 49.07 \\
\hline
\end{tabular}

${ }^{\mathrm{a}}$ All compounds were characterized and purity was assessed using ${ }^{1} \mathrm{H}$ NMR, MS and HPLC. 5- $\mathrm{HT}_{6} \mathrm{R}$ Receptor binding studies were carried out at Novascreen, USA. using Human recombinanat/HEK293 cells; Radioligand: $\left[{ }^{3} \mathrm{H}\right]$ LSD $(60-80 \mathrm{Ci} / \mathrm{mmol})$.

The most active compound $\mathbf{2 c}$ was further evaluated for its pharmacokinetic profile in male Wister rats and the results are given in Table-2. Compound $\mathbf{2 c}$ displayed good oral exposure with AUC $946 \pm 563 \mathrm{ng} \mathrm{h} / \mathrm{mL}$ and longer i.v. halflife $\left(\mathrm{t}_{1 / 2}=7.48 \pm 0.85 \mathrm{~h}\right)$ and oral bioavailability of $34 \%$. The brain and plasma levels of compound $\mathbf{2 c}$ were calculated in male Wister rats and it has shown high brain to plasma ratio of $13.8 \pm 2.6$.

Compound 2c was further evaluated for its cognitive retention in behavioural assay model like Morris water maze. It has significantly reversed the scopolamine induced memory deficit at $10 \mathrm{mg} / \mathrm{kg}$ p.o. dose in Morris water maze model, which was apparent from lesser target latency (Fig. 2).

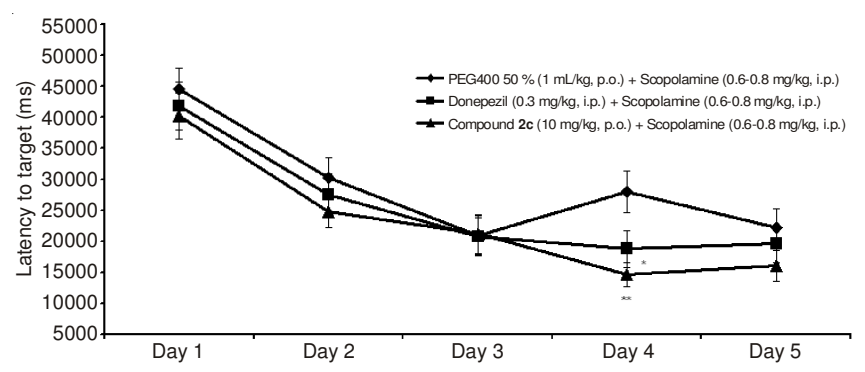

Fig. 2. Morris water maze test data for compound 2c in rats. Data represents Mean \pm SEM of latency to target, ${ }^{* *} p<0.01$, ${ }^{*} p<0.05$, (one way ANOVA, Dunnett's post hoc analysis)

\section{Conclusion}

In summary, a novel series of compounds $\mathbf{2}$ and $\mathbf{3}$ were designed by constraining the tryptamine side chain into a pyrrolidine ring through $\alpha$ and $\beta$ carbons and were found to have moderate affinities at $5-\mathrm{HT}_{6} \mathrm{R}$. The most active compound 2c has shown the acceptable pharmacokinetic profile along with the cognition potential at $10 \mathrm{mg} / \mathrm{kg}$ (Watermaze). The overall results indicated that these compounds have the potential to be $5-\mathrm{HT}_{6} \mathrm{R}$ ligands. Hence further efforts for their chiral separation and detailed profiling are under progress and constitute subject matter of our next publication.

\section{REFERENCES}

1. A. Meneses, G.P. Garcia, T. Ponce-Lopez and C. Castillo, Int. Rev. Neurobiol., 96, 27 (2011).

2. E.S. Mithchell, Int. Rev. Neurobiol., 96, 163 (2011).

3. K.G. Liu and A.J. Robichaud, Int. Rev. Neurobiol., 94, 1 (2010).

4. D. Witty, M. Ahmed and T.T. Chuang, Prog. Med. Chem., 48, 161 (2010).

5. G. Rossé and H. Schaffhauser, Curr. Topics Med. Chem., 10, 207 (2010).

6. D.J. Heal, S.L. Smith, A. Fisas, X. Codony and H. Buschmann, Pharmacol. Ther., 117, 207 (2008).

7. C.N. Johnson, M. Ahmed and N.D. Miller, Curr. Opin. Drug Dis. Dev., 1, 642 (2008).

8. Y. Tsai, M. Dukat, A. Slassi, N. MacLean, L. Demchyshyn, J.E. Savage, B.L. Roth, S. Hufesein, M. Lee and R.A. Glennon, Bioorg. Med. Chem. Lett., 10, 2295 (2000).

9. R.A. Glennon, M. Lee, J.B. Rangisetty, M. Dukat, B.L. Roth, J.E. Savage, A. McBride, L. Rauser, L. Hufesien and D.K.H. Lee, J. Med. Chem., 43, 1011 (2000).

10. A. Chuang, A. Foley and P.L. Pugh, Alzheimer's Dementia, 2, S631 (2006).

11. http://www.lundbeck.com/global (Accessed Jun 1, 2012).

12. R. Nirogi, V. Kandikere, K. Mudigonda, G. Bhyrapuneni and V. Jasti, Alzheimer's Dementia, 5, 250 (2009).

13. J. Bell, S.B. Bellaire, T. Leil, T. Comery, A. Plotka, J. Antinew, G. Vandal, S. Chalon and J. Kupiec, Alzheimer's Dementia, 7, S778 (2011).

14. N.V.S. Ramakrishna, K. Ramasastri, K. Prabhakar, K. Jagadishubabu, B. Thrinathreddy, G. Parandhama, A. Sobhanadri, G.P. Narasimhareddy, K.S. Anil, D.D. Amol, D. Adireddy, K.C. Anil and D. Pramodkumar, J. Enz. Inhib. Med. Chem., 27, 443 (2012).

15. A.D. Batcho and W. Leimgruber, Org. Synth., 63, 214 (1985).

16. R.V. Hoffman, Org. Synth., 60, 121 (1983). 